\title{
Nuevas tendencias en gestión de recursos humanos en las administraciones públicas: ¿están cambiando las reglas del juego?
}

\author{
Miquel Salvador Serna \\ Universidad Pompeu Fabra \\ miquel.salvador@upf.edu
}

\begin{abstract}
Resumen
Ante la importancia de los recursos bumanos, en el ámbito local se opta por la anulación de determinados valores propios de la gestión pública. Siguiendo la nueva gestión pública, la eficiencia y la productividad, asociadas a instrumentos como la evaluación del desarrollo profesional, pueden entrar en colisión al valorar las actuaciones de los empleados públicos. Cinco iniciativas configuran las nuevas tendencias en gestión de recursos bumanos en las administraciones públicas: la gestión por competencias, la evaluación de los recursos bumanos clásicos, la gestión del conocimiento, el desarrollo de sistemas de información y, finalmente, el rol de la comunicación interna.

Palabras clave: recursos bumanos, administración pública, gestión pública.
\end{abstract}

\begin{abstract}
Given the importance of Human Resources, the local administration has opted for the revocation of several values associated to public management. Following the New Public Management tradition, the stress on efficiency and productivity, associated to instruments such as the professional development evaluation, can compromise the value of public employees. Five initiatives compose the new tendencies in Human Resources Management in Public Administration: the Management Based on Competencies, the Classic Human Resources Evaluation, the Knowledge Management, the Information Systems Development, and finally, the Internal Communication Role.
\end{abstract}

Key-words: buman resources, public administration, public management. 


\section{Introducción}

En un contexto de referencias constantes a la necesidad y conveniencia de modernizar las administraciones públicas, con una amplia variedad de «modos» y de encabezamientos para intentar renovar el impulso transformador, el ámbito de los recursos humanos ha sido tradicionalmente bastante ignorado. Si bien últimamente se tiende a incluir en el discurso modernizador, a menudo se echan de menos concreciones que permitan valorar la opción real de cambio que se pretende.

En los últimos años se ha experimentado un cambio de tendencia, especialmente en el ámbito local. Ante la evidencia de la importancia de los recursos humanos, pero atendiendo a las dificultades para cambiar su gestión (o para afrontar su transformación), se recurre a una estrategia de beligerancia con determinados valores propios de la gestión pública. Se pretende, por diferentes vías y con diferentes mecanismos, superar el modelo burocrático que supuestamente caracteriza buena parte de nuestras administraciones.

Esta tendencia se concreta en prácticas que supuestamente favorecen una gestión más flexible, apoyadas en referentes teóricos de la denominada «nueva gestión pública» de inspiración anglosajona. El problema, sin embargo, es que no se importan solo instrumentos, como puede ser el caso de la evaluación del desarrollo profesional, sino que, de forma más o menos inconsciente, se incorporan también determinados valores y prácticas que van asociados a ellos. Esto supone, de facto, un cambio en el modelo de gestión pública y en lo que podríamos denominar «ecosistema» administrativo, que empieza a prestar atención a unos nuevos valores y principios sin una adecuada salvaguarda o garantía de mantenimiento de los que le eran propios y previos, y que justifican realmente su razón de ser. El énfasis exclusivo en la eficiencia y en la productividad, asociadas - como ya se ha mostrado - a instrumentos como la evaluación del desarrollo profesional, puede entrar en colisión al valorar determinadas actuaciones de los empleados públicos que tienen objetivos más difíciles de medir o a los que es preciso aplicar otros criterios valorativos.

La situación resulta especialmente lacerante en ámbitos como la gestión de los recursos humanos, que, si bien corresponde a la esfera interna de funcionamiento de las administraciones públicas, es el punto de partida de los procesos de socialización de sus miembros y un espacio donde se concretan valores públicos como la objetividad, la neutralidad o el mérito y se les da sentido. 


\section{Una aproximación analítica a las reglas de juego de la gestión de los recursos humanos de las administraciones públicas}

Los debates y la discusión sobre cómo repensar y mejorar la gestión de los recursos humanos en las administraciones públicas a menudo se han movido entre planteamientos excesivamente conceptuales o teóricos, cuando no retóricos, y planteamientos aplicados muy operativos en busca de respuestas a los problemas urgentes que hacía falta resolver. Si bien, obviamente, ambas aproximaciones son necesarias, muchas veces no se ha producido la necesaria integración para consolidar proyectos de reforma viables para transformar efectivamente los sistemas de gestión de recursos humanos en las administraciones públicas.

Así, al plantear el debate sobre las nuevas tendencias en gestión de recursos humanos, hay que prestar atención al desarrollo de estrategias, la incorporación de nuevas herramientas e instrumentos y el impulso de programas transformadores que, en la mayor parte de los casos, se han inspirado en referentes de la empresa privada o en referentes de otros países, con otras realidades sociales, económicas y administrativas. El problema es que en estos procesos de importación a menudo no se han considerado lo suficiente ni las premisas de partida ni los referentes conceptuales que subyacían tras buena parte de ese instrumental operativo.

Al considerar estos referentes salen a la luz las contribuciones realizadas en el mundo académico, desde diferentes disciplinas y con diferentes enfoques teóricos y analíticos. Si bien en este artículo no podemos hacer un repaso exhaustivo del complejo entramado conceptual vinculado a la gestión de personal en las administraciones públicas, sí que puede ser una buena ocasión para introducir algunos de estos enfoques, así como algunos de sus conceptos más destacados, para caracterizar la naturaleza y las dinámicas de transformación de este ámbito clave de la gestión pública.

Las denominadas "aproximaciones neoinstitucionalistas» que se proponen para apoyar el debate se basan en el concepto de «institución» o de «reglas del juego», entendidas como el resultado de un conjunto de normas, reglas, valores, estructuras y rutinas interrelacionadas, que definen las acciones que se consideran apropiadas en términos de relaciones entre el rol adoptado y la situación que se quiere afrontar, aportando estabilidad y significado a la conducta de los actores. Los actores utilizan este conjunto interrelacionado, que tiende a autorreforzarse, para determinar su conducta, es decir, para determinar qué y quién se incluye en el proceso de decisión, cómo se estructura la información, qué acciones se pueden tomar y qué consecuencias se consideran aceptables (Peters, 1999; Scott, 1995; March y Olsen, 1989). 
En estos enfoques, las instituciones se convierten en sistemas simbólicos, en ordenaciones de la realidad que la dotan de significado, creadoras de valores que condicionan las percepciones y la propia definición de los intereses de los individuos, incidiendo claramente en su comportamiento. A través de la interrelación social, las instituciones van adquiriendo racionalidad hasta alcanzar un nivel de consolidación -o institucionalización - que las convierte en parte objetiva e incuestionable de la realidad, todo ello como consecuencia de su carácter instrumental o su eficiencia ("mitos y ceremonias», como dicen Meyer y Rowan, 1991).

Para concretar el concepto, conviene describir los rasgos básicos que caracterizan la institución que preside la gestión de los recursos humanos en las administraciones públicas y que, genéricamente y más allá de los distintos vínculos laborales o diferencias de matices de las realidades administrativas concretas, podemos denominar «función pública».

Siguiendo la definición esbozada, la institución «función pública» viene regulada por un amplio abanico de normas, desde las referencias constitucionales (como los artículos 23, 28.1 y 103, referidos al acceso a la función pública y a sus principios rectores) a las leyes básicas del Estado, como la Ley de Bases de Funcionarios Civiles del Estado, de 20 de julio de 1963 (Decreto 315/1964, de 7 de febrero), la Ley 30/1984, de 2 de agosto, de Medidas Urgentes para la Reforma de la Función Pública, o el Estatuto Básico del Empleado Público, actualmente en debate. También cabe citar la normativa autonómica, como el Decreto Legislativo 1/1997, de 31 de octubre, por el cual se aprueba la refundición en un texto único de los preceptos de determinados textos legales vigentes en Cataluña en materia de función pública, o la normativa referida al ámbito local, como el Decreto 214/1990, de 30 de julio, por el cual se aprueba el reglamento del personal al servicio de las entidades locales, entre otras.

Además de este entramado jurídico, existe una amplia variedad de reglas informales que matizan la interpretación de sus principios. Pese a que no existen datos para poder ilustrar su concreción, varias investigaciones han destacado algunos de los rasgos más característicos de estos arreglos informales (Echevarría, 1995; Longo, 1995 y 2003), donde se reflejan más claramente algunos de los valores que caracterizan la gestión de los recursos humanos.

a) La idea de igualdad, muchas veces interpretada en términos de homogeneidad, especialmente en el acceso a los puestos de trabajo, a la carrera administrativa y en el sistema salarial y de incentivos.

b) El mérito y la capacidad como principios que regulan el acceso y el desarrollo de la carrera profesional, básicamente medidos a partir de su complementación formal. 
c) La unidad, a menudo asociada a la uniformidad en la configuración del sistema de función pública (clasificación de los empleados, reclutamiento, remuneración, estructura de puestos de trabajo).

d) La centralización de la gestión y la administración de las principales actividades vinculadas a la gestión de recursos humanos, tanto en la relación entre Estado, comunidades autónomas y entes locales como en el seno de las respectivas administraciones.

e) La jerarquía como principal mecanismo de coordinación entre unidades, aunque matizada por el surgimiento de regímenes singulares que favorecen un sistema más próximo a una red que a una estricta pirámide.

Los rasgos apuntados se relacionan con la creciente fragmentación que caracteriza al empleo público, atendiendo tanto a la consolidación de colectivos profesionales con regulaciones específicas como al creciente peso de los procesos de externalización y la aparición de un amplio abanico de figuras organizativas que gestionan, con diferentes grados de autonomía, sus recursos humanos. Con todo, resulta interesante observar cómo, pese a ser en estos espacios más "externos» donde aparecen las iniciativas de cambio aparentemente más fuertes, sus impactos efectivos acaban siendo bastante limitados, de manera que, de una u otra forma, tienden a reproducirse buena parte de las pautas de funcionamiento de la función "pública» que se han definido.

La aplicación de la normativa formal, a partir de los valores y arreglos informales comentados, ha generado una serie de rutinas y procedimientos que también se han reflejado en la creación de una estructura orgánica alrededor de la gestión de recursos humanos. Ambos elementos resultan especialmente relevantes en la medida en que constituyen tanto un referente simbólico de la importancia otorgada a este ámbito como un canal de formación y socialización en el trabajo que, a menudo, supera las carencias de los programas formativos más formalizados, aun cuando no necesariamente en la línea perseguida por la dirección de la organización.

Para interpretar las interrelaciones de estos elementos hay que prestar atención a la separación entre política y función pública. Desde la configuración inicial de buena parte de nuestras administraciones públicas democráticas, el ámbito de la gestión de los recursos humanos se asimiló a una esfera de carácter técnico, asociada a una visión de poca "rentabilidad política», tanto por su poca visibilidad externa como por las dificultades para obtener resultados a corto plazo. Esto propició que las máximas unidades directivas de función pública no fueran un espacio especialmente atractivo para muchos líderes políticos, y se convirtieran en "un lugar de paso» en sus carreras. El alejamiento del debate público, y especialmente del discurso y la práctica, favoreció que la discusión sobre la configuración de la función pública permaneciera en círculos 
especializados, y que fuera en ellos donde se formularan alternativas y propuestas de reforma del sistema de gestión de recursos humanos.

En cualquier caso, de la interrelación de estos elementos surgen unas «reglas del juego» que constriñen y dirigen la actividad de los agentes implicados en «la función pública», a menudo no tanto de forma explícita como a través de la definición de marcos de referencia que se toman como dados y que no cuestionan.

Esta institución tiene reflejo tanto en el ámbito concreto como en el genérico. En un plano más operativo, se asocia a su vigencia en determinados procedimientos vinculados a los procesos selectivos (y la no exploración de nuevas fórmulas o de nuevos equilibrios entre las fases o componentes de estos), a la linealidad de las retribuciones (incluso en los componentes retributivos planteados como variables), a la no aplicación efectiva del régimen disciplinario o a una formación interna concebida más como un premio al empleado que como un instrumento de gestión al servicio de las necesidades de la organización. En el ámbito genérico, este marco de referencia se traduce en un acusado escepticismo en relación con las posibilidades de transformar el modelo vigente, o en determinadas críticas a propuestas formuladas externamente o desde la dirección política de la organización.

Partiendo de este enfoque, para una correcta gestión de las personas que trabajan en la Administración resulta clave conocer las normas, pero también saber interpretarlas, identificando los valores que las orientan y los procesos y estructuras en que se sustentan. Dadas estas premisas, lo importante no es tanto establecer incentivos y obligaciones puntuales a los individuos, sino averiguar las interacciones de los componentes de la institución para facilitar su transformación, de acuerdo con las dinámicas de funcionamiento efectivo de las administraciones públicas.

\section{Un enfoque estratégico para cambiar las "reglas del juego»: política, gestión y administración de recursos humanos}

Para poder avanzar en esta línea, es preciso contar con un planteamiento estratégico coherente con los objetivos de la organización. En este sentido, resulta útil recuperar la distinción entre los niveles de política, gestión y administración de los recursos humanos, atendiendo al carácter estratégico con que se diseñan y se coordinan las actuaciones en este ámbito.

El primer nivel, correspondiente a las políticas de personal en las administraciones públicas, se vincula al establecimiento de los objetivos estratégicos que expresan equilibrios de valores en un contexto económico, social, político y tecnológico determinado, 
y que se desagregan en objetivos operativos formulados mediante normas, directivas y resoluciones, cuyo cumplimiento corresponde a los responsables de las diferentes actividades de personal, es decir, a los responsables de la gestión y administración de personal.

Para decidir estos objetivos estratégicos, los responsables de diseñar la política de personal precisan del apoyo de los gestores de recursos humanos, encargados de plantear los problemas y las posibles soluciones en términos de recursos y condicionamientos, de objetivos y de valores de la misma organización. Es en este sentido que la gestión de personal no se limita a organizar, dotar, dirigir y controlar, sino también a equilibrar los sistemas técnicos y sociales de la organización con los recursos y los condicionantes de su entorno, con la finalidad de alcanzar los objetivos estratégicos establecidos. Lo que transforma las técnicas y los instrumentos de personal en una verdadera gestión de recursos humanos es el hecho de estar al servicio de una política de personal, que es preciso concebir integrada en una estrategia global de desarrollo de la organización.

La administración del personal, el tercer nivel de la «cadena» política-gestión-administración, empieza cuando se acaba la gestión de personal, es decir, cuando desaparece la responsabilidad para tomar decisiones sobre objetivos operativos y sobre cómo alcanzarlos. La administración de personal no supone tomar decisiones, sino aplicar unas técnicas predeterminadas con las cuales los gestores de personal consideran que se podrán cumplir los objetivos prefijados.

De esta definición se desprende que, en el supuesto de que se rompa la "cadena»y, por ejemplo, la gestión de personal se base en la simple aplicación de regulaciones y en la aplicación mecánica de técnicas, entonces ya no se puede hablar de "gestión» en los términos definidos, sino de una mera "administración de personal».

Así, una Administración Pública sin política de personal — consecuentemente, sin gestión de personal y solo con administración de personal- se convierte en una organización que solo puede reproducir el modelo organizativo del que parte, incapaz de adaptarse a las transformaciones de su entorno.

En la realidad de muchas administraciones de nuestro entorno de referencia, se echa de menos la definición de una clara política de personal conectada a la estrategia de la organización, a menudo por ausencia de esta última y por la inexistencia de objetivos específicos. Obviamente, si la organización no tiene claramente definidos sus objetivos y la estrategia para alcanzarlos, difícilmente podrá establecer una política de personal coherente con ellos. Pero incluso en aquellas organizaciones públicas donde se han llegado a definir marcos de actuación y objetivos concretos, así como una estrategia para lograrlos, el ámbito de los recursos humanos tampoco llega a conectarse lo suficiente claramente. 
Esta situación debe asociarse a la tradicional consideración de la gestión de personal como un ámbito reservado a especialistas y conocedores del complejo entramado de normas e instrumentos de gestión que lo caracteriza. Esto ha propiciado su distanciamiento del debate público y del interés de los políticos, que han tendido a desentenderse de él apelando al funcionamiento rutinario del aparato administrativo. Este distanciamiento, que a menudo se traduce en falta de apoyo político a iniciativas que podrían suponer conflictos potenciales, ha dificultado — cuando no ha impedido directamente - tanto la propia definición como la puesta en marcha de iniciativas transformadoras de mayor alcance.

En este caso, sin embargo, se trata de un síntoma de una enfermedad muy importante que, además de impedir el surgimiento y la consolidación de determinadas iniciativas, debilita de forma notable las capacidades organizativas para facilitar su adecuación a los cambios que se producen en su entorno. Así, más allá de centrar la atención y los esfuerzos en impulsar determinadas iniciativas puntuales, lo que hay que hacer previamente es consolidar una verdadera política y gestión de los recursos humanos en el seno de la organización.

\section{Nuevas tendencias en gestión de recursos humanos}

La carencia de claras orientaciones estratégicas en la política de recursos humanos en buena parte de nuestras administraciones, a la que nos hemos referido en el apartado anterior, no ha sido impedimento para que muchas de ellas emprendieran interesantes iniciativas transformadoras.

Pese a que la normativa estatal y autonómica se ha utilizado a menudo como argumento para explicar las dificultades para transformar la institución, lo cierto es que no se ha agotado el potencial de cambio que permite su interpretación. Precisamente, las iniciativas que se exponen a continuación se han basado en la interpretación de esos preceptos para incorporar herramientas procedentes de otros contextos de referencia.

Así, sin ánimo de exhaustividad, a continuación se hace mención de cinco iniciativas que configuran algunas de las nuevas tendencias en gestión de recursos humanos en las administraciones públicas.

\subsection{La introducción de la gestión por competenciasł replanteando la lógica de puestos de trabajo}

Una de las iniciativas clave del proceso de reforma de la función pública estatal que tomó fuerza especialmente a partir de la Ley 30/1984, de 2 de agosto, de Medidas 
Urgentes para la Reforma de la Función Pública, fue la estrategia anticorporativa que pretendía racionalizar el aparato burocrático estatal conformado por cuerpos de funcionarios y someterlo a la nueva dirección política. Uno de los instrumentos clave en este proceso fue el impulso de un modelo de función pública basado en el concepto de "puesto de trabajo», concretado en la elaboración y aprobación de relaciones de puestos de trabajo (RLT) como instrumento de ordenación de personal y pieza clave para estructurar la carrera administrativa y el sistema retributivo. Este instrumento se extendió rápidamente tanto a las entonces incipientes administraciones autonómicas como a las locales.

Una idea asociada a este impulso era la normalización y homogeneización del empleo público, que buscaba elementos objetivos para estructurar y dar coherencia a unas administraciones públicas en fase de crecimiento. De este modo, la misión y los objetivos de la organización se pretendían traducir en tareas agrupadas alrededor de puestos de trabajo, como punto de partida para el diseño de estructuras orgánicas racionales y adecuadas a las actividades que debían desarrollar. Las herramientas para hacer operativos el análisis, la definición y la valoración de puestos de trabajo se diseñaron siguiendo el modelo desarrollado por una importante empresa de consultoría, copiado de forma mimética, con apenas ligeras adaptaciones, para la práctica totalidad de administraciones públicas españolas.

Aun así, en la práctica se mantuvieron muchos de los elementos propios de la lógica de cuerpos, especialmente en ámbitos tan importantes como el acceso y la promoción interna. Desde el punto de vista cultural, al mismo tiempo, se mantuvieron las tensiones de determinados colectivos profesionales (Ferret, 1997).

Así, tanto la vigencia de principios propios de la lógica de cuerpos como la utilización laxa de la definición de puestos de trabajo, muy a menudo ajustada a las necesidades de flexibilidad y adaptación que requería una gestión de recursos humanos con dificultades para retribuir $y$ promocionar a sus efectivos, favorecieron el cuestionamiento del modelo. Este cuestionamiento implícito se concretaba en una falta de adaptación entre una realidad formal de estructuras basadas en puestos de trabajo y una gestión real que las utilizaba solo como legitimación a posteriori de decisiones tomadas mediante criterios alejados de la racionalidad a la que, supuestamente, respondían aquellos instrumentos.

A la vista de esta situación, una de las nuevas tendencias para intentar mejorar la adecuación de elementos propios de la estructura orgánica, como son los puestos de trabajo, y elementos propios de la gestión de los recursos humanos, como son los perfiles profesionales de los efectivos de la organización, ha sido el impulso de la denominada "gestión por competencias». 
Más allá de este punto de partida, lo realmente destacado de la gestión por competencias es el hecho de proponer un modelo de gestión integral de los recursos humanos que parte de la identificación y el desarrollo de las competencias profesionales fundamentales para el desempeño de un puesto de trabajo. Diversas administraciones, como el Ayuntamiento de Manlleu y la Diputación de Barcelona, o empresas públicas como BSM (Barcelona Servicios Municipales) están poniendo en marcha sistemas de gestión por competencias.

La promoción de este modelo pretende superar la rigidez del modelo actual de puestos de trabajo para centrar la atención en los profesionales que integran la organización, identificando las competencias definidas en términos de capacidades, habilidades y conocimientos, y que, a la vez, se agrupan en actitudes y aptitudes para el desarrollo de la actividad.

Su concreción parte de un análisis de las competencias de los puestos de trabajo en que se describen las requeridas para desarrollar las funciones asociadas, y que se contrasta con una evaluación de las competencias individuales de que dispone cada persona de la organización. El contraste de las diferencias entre las competencias que posee cada individuo y las que requiere el puesto aporta las pautas para la gestión de los procesos de selección, promoción interna, formación y evaluación del desempeño.

Dado el poco tiempo que llevan en marcha estas iniciativas, resulta muy arriesgado hacer un balance de ellas; de lo que no hay duda, en cualquier caso, es de que representan un importante elemento dinamizador para dar coherencia al conjunto de subsistemas de la función de personal. Aun así, como ya se ha apuntado en un apartado anterior, su adecuada concreción requiere de una clara explicitación de la misión y los objetivos que debe lograr la organización, traducibles en competencias profesionales asociadas, sin las cuales el modelo corre el riesgo de convertirse en otro nivel formal añadido a los ya existentes y que están más o menos desconectados de la realidad de la gestión de recursos humanos.

\subsection{La evaluación de los recursos humanosł un clásico todavía muy poco extendido}

Una iniciativa con mayor tradición en el mundo de la función pública en nuestras administraciones es la denominada "evaluación del desarrollo»o "evaluación de los recursos humanos». Ya en la primera mitad de la década de 1990 aparecieron las primeras iniciativas en materia de evaluación en diferentes administraciones catalanas, como, por ejemplo, la Diputación de Barcelona, la Generalitat de Catalunya, la Universidad 
Autónoma de Barcelona o la Universidad Politécnica de Cataluña (en estos dos últimos casos, en relación con el colectivo de personal de administración y servicios).

Con ligeros matices, todas estas experiencias han formulado sistemas de evaluación del desarrollo en términos de proceso continuo, sistemático, orgánico y en cascada, de expresión de juicios alrededor del personal de una organización; un proceso de expresión de juicios en relación tanto con el trabajo habitual de cada empleado como con su potencial de desarrollo, y que se planteaba con una óptica histórica y prospectiva que pretendía integrar los objetivos de la organización con los del individuo (Salvador, 1997).

La mayor parte de estas iniciativas, aunque con diferentes denominaciones, orientaciones y alcance, planteaba la introducción de la evaluación del desarrollo como una herramienta para fomentar una visión integral de la gestión de los recursos humanos. Entre otras cuestiones, se destacaba su potencial para apoyar las actuaciones en materia de retribuciones y, concretamente, la gestión del complemento de productividad, en la carrera administrativa o en el establecimiento de acciones formativas a partir de las carencias detectadas. En otras experiencias se pretendía destacar su potencial como instrumento de diálogo entre los mandos y sus colaboradores, para sentar las bases de un modelo de dirección por objetivos.

Estos rasgos compartidos por la mayor parte de las experiencias constituyen solo un punto de partida, que se concretó en diferentes estrategias e instrumentos, también con diferentes resultados. Sin querer extendernos demasiado en la descripción de estas experiencias, vale la pena destacar que, si en el caso de la Diputación de Barcelona la experiencia se implementó para apoyar el desarrollo de la progresión profesional denominada "carrera universal», en la Generalitat de Catalunya su aplicación se diluyó y se acabó convirtiendo en un instrumento meramente formal para distribuir un complemento a finales de año con carácter básicamente lineal. En las dos experiencias en administraciones universitarias, la iniciativa dejó de aplicarse, aunque sirvió de base para otras iniciativas de racionalización de la gestión interna (como, por ejemplo, la dirección por objetivos).

El balance de estas experiencias viene a reforzar la idea de que la aplicación de la evaluación del desarrollo profesional en las administraciones públicas requiere de una adecuada adaptación, especialmente teniendo en cuenta las reglas del juego que caracterizan la gestión de los recursos humanos. Elementos como la resistencia al cambio o los procesos de desajuste entre la formalización de determinadas actividades y su impacto y valores reales, son solo algunos de los aspectos que es preciso considerar si se pretende introducir una cultura orientada a crear valor, al trabajo por objetivos, a la responsabilidad para la obtención de resultados o a la alineación de los retos profesionales personales con los de la organización. 
Desde esta perspectiva, por tanto, la evaluación del desarrollo profesional representa algo más que una mera herramienta de gestión de los recursos humanos, pues incorpora un potencial de apoyo a los procesos de cambio organizativo, que incide tanto en la cultura administrativa - a través de la comunicación que propicia - como en las rutinas de trabajo por objetivos y su revisión, y, al mismo tiempo, ofrece una importante base de información para validar la evolución del sistema en su conjunto.

Este potencial ha propiciado que, pese a que algunas experiencias no hayan sido lo suficientemente exitosas, la evaluación del desarrollo profesional continúe siendo un tema central en los debates sobre la gestión de los recursos humanos en las administraciones públicas. Así, además de las experiencias citadas, han surgido iniciativas en otras administraciones. En este sentido, resulta significativa la referencia a ella en la mayor parte de los debates que se han articulado tanto en el ámbito catalán (como, por ejemplo, en torno al Libro Blanco de la Función Pública de Cataluña) como en el ámbito estatal (en el proceso de elaboración del actual Estatuto Básico del Empleado Público, en el capítulo IX sobre «la promoción profesional de los empleados públicos»).

Para superar las dificultades, tanto de carácter operativo y cultural como de resistencia por parte de diferentes colectivos, hay que plantear el sistema de evaluación no solo como un instrumento más, sino como un elemento de cambio integral del sistema de gestión de recursos humanos. En este sentido, las experiencias de éxito recuerdan que hay que vincular la evaluación del desarrollo con la política y la gestión de los recursos humanos, y estas con la estrategia global de la Administración. Asimismo, hay que atender a los diferentes parámetros de concreción de la evaluación, tanto respecto al planteamiento y los objetivos como respecto a sus aplicaciones, a la caracterización del instrumento y, especialmente, a la estrategia de implementación.

\subsection{La gestión del conocimiento: las bases para crear organizaciones que aprendan}

Por "gestión del conocimiento" se entiende el proceso a través del cual las organizaciones recopilan, sistematizan y difunden el conocimiento que les es útil para mejorar su actividad. Tanto por la amplia diversidad de fuentes y formatos en que se puede encontrar la información y el conocimiento como por la multiplicidad de actores implicados y, especialmente, por los valores y el cambio cultural que suponen, las iniciativas emprendidas en este ámbito son todavía muy incipientes en las administraciones públicas.

Como fuentes de conocimiento, a menudo se destacan las de carácter formal y más «tangible», como la normativa, los informes y los manuales, plasmados en diferentes 
formatos y soportes, y las de carácter más informal o, cuando menos, menos tangibles, como las experiencias y habilidades desarrolladas por cada profesional en el ejercicio de sus funciones. Estas últimas, si bien a menudo no se presentan de forma sistemática, acaban siendo las más importantes tanto en las actividades habituales como en los procesos de aprendizaje en las funciones específicas del puesto de trabajo (a menudo mucho más que los cursos de formación).

La idea central en las iniciativas de gestión del conocimiento es recopilar el conocimiento que "fluye» por la organización, sistematizarlo para aprovechar su verdadero potencial agregado y ponerlo al alcance de los diferentes agentes implicados; en otros términos, "gestionarlo».

Desde el punto de vista operativo, las iniciativas de gestión del conocimiento combinan diferentes metodologías para:

a) identificar las fuentes y los flujos de conocimiento con mayor valor añadido para la organización;

b) organizar esas fuentes y flujos de acuerdo con los objetivos que se plantean priorizar, sistematizando contenidos para hacerlos útiles y valiosos para los diferentes destinatarios;

c) difundirlos entre los colectivos a los que pueden aportar un valor añadido para mejorar su actividad;

d) $y$, finalmente, crear valor poniendo al alcance del resto de la organización tanto los contenidos como, y especialmente, las dinámicas de aprendizaje y gestión del conocimiento emprendidas.

Aunque no hay que asociar exclusivamente el impulso de las iniciativas de gestión del conocimiento al uso de las tecnologías de la información y la comunicación, a menudo estas se han utilizado para hacerlas operativas. Por otra parte, las pocas referencias que se encuentran en este campo en el ámbito de las administraciones públicas catalanas se basan precisamente en el uso de aplicaciones tecnológicas específicas.

Entre estas contadas experiencias hay destacar la iniciativa "Poliedro», impulsada en la Universidad Politécnica de Cataluña, y el aplicativo «BSCW» («Basic Suport for Collaborative Work»), desarrollado en la Generalitat de Catalunya. Lo limitado del número y, también - especialmente en el segundo caso-, del impacto efectivo de estas iniciativas debe atribuirse a la falta de una estrategia de cambio cultural que acompañe la puesta en marcha de la propuesta. De nuevo, el desarrollo de una iniciativa en materia de gestión de recursos humanos descontextualizada de una política integral que le dé sentido puede dar ciertos resultados positivos, pero a menudo se queda muy lejos de su potencial de transformación y mejora. 
Por las mismas dinámicas de funcionamiento de las administraciones y la creación de determinadas rutinas informales, en muchas organizaciones públicas ( $y$ también privadas) se ha visto con bastante desconfianza el hecho de poner al alcance de la organización determinados conocimientos informales, tanto por lo que implican de transparencia y apertura a posibles críticas como por la posibilidad de "hacerse» prescindible tras la cesión de un "recurso» estratégico como es el propio conocimiento de la actividad. El necesario liderazgo para impulsar un cambio cultural que modifique el tipo de relaciones de poder, formales e informales, favoreciendo dinámicas para compartir recursos y de mayor implicación en un proyecto común, a menudo no se ha dado o, al menos, no con la suficiente fuerza y durante el suficiente tiempo como para permitir esa transformación. En otros términos, no se ha conseguido cambiar las «reglas de juego» o las instituciones vigentes.

\subsection{El desarrollo de sistemas de información: el apoyo a la toma de decisiones y a su evaluación}

Pese a que las administraciones públicas disponen desde hace bastante tiempo de diversos centros dedicados a la recogida de datos sobre sus efectivos de personal, desde el propio registro de personal hasta las unidades de nóminas, todavía hoy resulta muy escasa la utilización efectiva de su potencial de información tanto para apoyar la toma de decisiones como para dar cuenta de la evaluación del impacto de las decisiones tomadas en el pasado.

Las administraciones deberán ser capaces de centralizar y transformar la acumulación de datos individualizados de sus profesionales en información agregada y útil, y también en conocimiento, para introducir racionalidad o, cuando menos, mayor evidencia empírica, en el proceso de análisis de la realidad actual, en la elaboración de diagnósticos y en la toma de decisiones en el ámbito de gestión de recursos humanos.

Esta situación se puede asociar a la propia concepción de las unidades encargadas de recopilar información, a menudo presentadas como centros donde recopilar historiales, donde "dar fe» de los datos recogidos sobre la vida laboral de cada empleado público, sin incorporar un planteamiento de gestión orientado a optimizar el potencial de su explotación agregada para apoyar a la dirección. Además, los profesionales encargados de estas unidades no han recibido ni el apoyo, en términos de recursos y de formación, ni las directrices claras para facilitar un verdadero replanteamiento de la razón de ser de su ámbito.

Pese a que hemos iniciado el comentario de esta tendencia con la diagnosis de la realidad más extendida, hay que destacar la existencia de experiencias innovadoras en 
este ámbito, algunas de ellas con cierta trayectoria. A modo de ejemplo, la Dirección General de la Función Pública de la Generalitat impulsó, a mediados de la década de 1990, la elaboración de sistemas de información con un sugerente título («Indicadores de impacto de las políticas de personal de la Generalitat de Catalunya»).

Con el apoyo de agentes externos, su desarrollo combinó la integración de las diversas fuentes de información sobre personal dispersas en la organización, desde la base de datos centralizada de personal (Gestión Integrada de Personal-Sistema Integrado Personal o GIP-SIP) a las diferentes bases de datos desarrolladas por los distintos departamentos, así como las recogidas a nivel sectorial por los diferentes centros de formación (por ejemplo, la Escuela de Administración Pública de Cataluña, el Centro de Estudios Jurídicos y Formación Especializada o la Escuela de Policía de Cataluña). El desarrollo de este sistema integrado de información se complementó con la realización de una encuesta de cultura organizativa y clima laboral (una de las pocas experiencias conocidas en el seno de nuestras administraciones), así como con la realización de diversos grupos de discusión para, desde un punto de vista cualitativo, explorar argumentos y contrastar diagnosis con diferentes perfiles profesionales. Desgraciadamente, la experiencia se prolongó durante seis años y posteriormente decayó, si bien la vertiente de integración de bases de datos ha tenido cierta continuidad, hasta el punto de que hoy se ha convertido en un referente a la hora de definir un apartado orientado a los directivos dentro la intranet de la Administración autonómica.

Con la introducción de las tecnologías de la información y la comunicación y, en especial, al redefinir los roles de los diferentes agentes implicados en la introducción y gestión de datos de personal, se abren nuevas fronteras en este ámbito clave de apoyo a la gestión integrada de recursos humanos.

Tradicionalmente, la introducción de los datos en el registro de personal, así como su explotación posterior, estaban en manos de unidades específicas a menudo caracterizadas por un alto componente técnico asociado a la gestión de las complejas aplicaciones utilizadas. Si bien en muchos de casos esta situación se mantiene, resulta interesante observar cómo, gracias a la innovación tecnológica y, en especial, a la extensión de portales del empleado que se utilizan como plataformas de gestión de diversos trámites de personal, tanto la introducción como la posible explotación de los datos (obviamente salvaguardando las necesarias cautelas en cuanto a protección de datos individuales), resulta un ámbito más abierto y que trasciende aquellas unidades originales. Así las cosas, si bien se mantiene la necesidad de un gestor único de la base de datos de personal encargado de su mantenimiento y depuración, el número de agentes con capacidad para interactuar con esta, tanto con respecto a la introducción de datos como, potencialmente, a su extracción y explotación, parece multiplicarse. 
Aun así, y como acostumbra a pasar con muchos de los avances en el campo de la tecnología, no basta contar con el instrumento, sino que también se precisa una adecuada concienciación y aprendizaje para optimizar su utilización. Mejorar este sistema debería ser una de las tareas importantes de la unidad de gestión de recursos humanos, mas no solo para disponer de un mejor instrumento para almacenar la información, sino para convertirla en conocimiento útil. Dar este paso no es una cuestión de inversión en equipamiento informático (ni de hardware ni de software), sino de formación y reciclaje, de cambio de perfiles de los profesionales que trabajan con las bases de datos relacionadas con la gestión de personal, pero también de cambio en los usuarios o potenciales destinatarios de sus explotaciones.

Potenciar que los directivos trabajen con mejores niveles de información, con datos concretos que permitan afinar sus diagnosis, es un elemento esencial para mejorar la toma de decisiones. De poco servirá la gestión de la información si no se acompaña de un cambio en los hábitos con respecto a su utilización. En este proceso, uno de los cambios fundamentales es de carácter cultural y se refiere a la capacitación de muchos de los directivos públicos para que se acostumbren a trabajar con datos, tanto en la toma de decisiones como en el seguimiento de las acciones emprendidas y su posterior evaluación. En otras palabras, es preciso cambiar el estilo de hacer las cosas y, de nuevo, las reglas del juego en la toma de decisiones vinculadas a la gestión de los recursos humanos.

\subsection{La comunicación interna y el rol de las intranets corporativas}

Tradicionalmente se han desarrollado, con mayor o menor orientación estratégica, diferentes instrumentos de comunicación entre la organización pública — su direccióny el conjunto de empleados que la integran.

Bastantes administraciones acostumbran a entregar un manual de acogida a los empleados recién incorporados. A menudo en forma de publicación, en el manual se exponen de manera sintética la historia, las actividades, los valores y el funcionamiento de la organización; se presenta, pues, como un instrumento que pretende facilitar la integración del nuevo personal. Otro instrumento clásico dirigido al conjunto del personal son las revistas de comunicación interna, publicaciones periódicas de difusión interna que ofrecen un tratamiento más o menos periodístico de informaciones sobre las actividades propias de la organización, así como de temas generales que añaden interés a los contenidos.

Aunque sin llegar a sustituir en muchos casos a estos instrumentos clásicos de información interna, una nueva tendencia en la gestión de recursos humanos ha sido la 
proliferación de intranets corporativas y los denominados "portales del empleado». Estas redes de distribución de información accesibles solo a los trabajadores de la organización han sido el canal de comunicación que más ha crecido en los últimos años, de manera que la mayoría de las grandes administraciones públicas cuentan ya con esta estructura. Así, las encontramos en los diferentes departamentos de la Generalitat de Catalunya, en la Diputación de Barcelona y también en el Ayuntamiento de Barcelona, por citar algunos ejemplos. A menudo estos instrumentos han integrado muchos de los antiguos canales de comunicación en un mismo contenedor: buzón de sugerencias, tablón de anuncios, encuestas de opinión, notas informativas, noticias de actualidad, boletines, entre otras.

En algunas administraciones, este instrumento también se ha planteado como una plataforma para gestionar determinados trámites vinculados a la gestión de personal, como la solicitud de permisos, el acceso a cursos de formación, consultas y gestiones vinculadas a la nómina, entre otros. En el caso de la Generalitat, por ejemplo, el portal EPOCA (Empleado Público On-line de Cataluña) se ha concebido siguiendo este planteamiento. En el apartado «Recursos humanos» de la intranet del Ayuntamiento de Barcelona hay un bloque dedicado a "trámites» con ese mismo enfoque.

Resulta interesante observar cómo, a menudo, estos canales de comunicación interna y de gestión no hacen sino transferir en un nuevo soporte muchos de los rasgos distintivos del enfoque de recursos humanos de las organizaciones que representan. Así, pese a los diseños más o menos acertados, con contenidos variables y aplicaciones de gestión más o menos compartidas, lo que sí los diferencia es la manera como plantean la relación entre la organización y los profesionales que la integran. Si bien se han extendido los instrumentos orientados a favorecer la interacción, si no se consigue generar confianza e implicación por otras vías, la participación efectiva de los profesionales quedará bastante lejos del potencial que aportan las tecnologías.

Así, los avances en la transparencia, la mayor agilidad en la gestión de determinados trámites o la mejora en la información disponible son solo algunas ganancias potenciales que precisan de cambios en las actitudes y en los procesos internos de la organización y que van más allá de la mera aplicación de tecnología.

En otros términos, si no se han conseguido cambiar las reglas de juego que rigen las relaciones entre los profesionales y la organización, con la introducción de este nuevo instrumento solo se conseguirán ganancias incrementadas que quedarán lejos de su potencial efectivo. 


\section{Algunos comentarios a modo de conclusión: el ajuste entre las instituciones y las nuevas tendencias en gestión de recursos humanos}

Las cinco iniciativas destacadas, sin ánimo de exhaustividad, permiten mostrar dos ámbitos clave en los que se está trabajando para transformar la gestión de los recursos humanos: el desarrollo de una visión integral del sistema y la revalorización de los intangibles.

Los diferentes ejemplos comentados pretenden aportar herramientas que obedecen a la necesidad de desarrollar una visión integral del ámbito de la gestión de recursos humanos que aporte respuestas que vayan más allá de la actuación puntual en alguno de los ámbitos que constituyen el sistema (como la selección o la retribución, por ejemplo). Además, todas ellas apuestan claramente por otorgar un papel clave a los factores intangibles, como el conocimiento, las competencias, la información o la comunicación, reconociendo que en ellos radica buena parte del potencial efectivo de la organización para implicar a los profesionales que la integran.

Otro rasgo compartido por las cinco iniciativas comentadas es el empleo de las tecnologías de la información y la comunicación como pieza clave para su concreción y operatividad, si bien estas solo constituyen un medio de apoyo al desarrollo de una aproximación específica a los recursos humanos.

El todavía limitado alcance de algunas de estas iniciativas, así como los pobres resultados alcanzados por algunas de las que tienen mayor trayectoria temporal, hay que buscarlos en su estrategia de adaptación a la realidad institucional que pretenden transformar.

La falta de conexión entre lo que presuponen esas recetas y la realidad de las normas, valores, estructuras y procesos que configuran las reglas del juego y la «manera de hacer» de la gestión de personal en nuestras administraciones son la principal dificultad para su aplicación efectiva y para un adecuado aprovechamiento del potencial que ofrecen.

Articular normas ya sabemos, aunque siempre hay margen para la mejora $y$, tal vez, haría falta innovar en algunas de las vigentes (pese a las limitaciones de la Administración General del Estado). En cualquier caso, lo que se ha querido destacar es la necesidad de invertir especialmente en la transformación de los valores, las estructuras y los procesos. Es en estos ámbitos donde hay más libertad de movimientos y donde, a la vez, los avances pueden dar lugar a mejoras muy notables y sostenibles a lo largo del tiempo.

Con esto queremos aludir a la necesidad de impulsar un "nuevo estilo» de gestionar los recursos humanos, una nueva manera de hacer las cosas que, a la vez, facilite el 
que este ámbito pase a formar parte de las prioridades de los máximos niveles directivos y no sea una dimensión donde se dé por hecho que se puede hacer poco y no se puede esperar demasiado.

Identificar las instituciones vigentes en las administraciones públicas se muestra, desde este enfoque, como un paso previo para valorar la oportunidad de incorporar nuevas herramientas de gestión. Para hacerlo hay que observar la legislación, pero también cómo se aplica; hay que observar las estructuras orgánicas y los procedimientos administrativos, pero también la cultura y los valores que orientan su funcionamiento. Ahora bien, más allá de estos elementos, hay que conocer sus interrelaciones de refuerzo mutuo, a través de las cuales las instituciones tienden a reproducirse sin demasiadas modificaciones. Se trata, en definitiva, de conocernos algo mejor a nosotros mismos antes de implantar soluciones descontextualizadas, provengan estas de otras administraciones públicas o directamente del sector privado.

\section{Bibliografía}

Dowding, K. (1995): The Civil Service. Routledge, Londres.

Ferret, J. (1997): «El sistema español de formación y selección de altos funcionarios». En: Sánchez Rodríguez, A.; Núñez Santos, M.* T. (eds.): La función pública a debate. Actas del I Congreso Nacional de Función Pública. Fundación Genesian y Fundación Educación y Futuro, Sevilla.

Longo, F. (2003): "Revisió d’alguns elements clau de la situació de la funció pública a Catalunya». En Fernández, A.; Muñoz, X.; Cisternas, X. (coord.): Informe Pi i Sunyer sobre l'Administració de la Generalitat de Catalunya. Fundació Carles Pi i Sunyer, Barcelona.

Longo, F. (1995): "Reforma del empleo público: tótem y tabú», Gestión y Análisis de Políticas Públicas, n. ${ }^{\circ}$ 2, enero-abril.

March, J. G.; Olsen, J. P. (1989): Rediscovering Institutions. Free Press, New York.

Peters, B. G. (1999): Institutional Theory in Political Science: The "New Institutionalism». Painter, Londres.

Salvador, M. (1997): Politiques de funció pública: l'avaluació de recursos bumans. Escuela de Administración Pública de Cataluña, Barcelona.

Sсотт, W. R. (1995): Institutions and Organizations. Sage, Thousand Oaks. 